Article

\title{
Two-Tier Care Pathways for Liver Fibrosis Associated to Non-Alcoholic Fatty Liver Disease in HIV Mono-Infected Patients
}

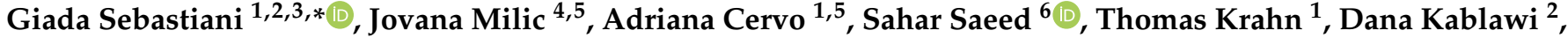 \\ Al Shaima Al Hinai ${ }^{7}$, Bertrand Lebouché ${ }^{1,3,8}{ }^{\mathbb{D}}$, Philip Wong ${ }^{2}$, Marc Deschenes ${ }^{2}$, Claudia Gioè ${ }^{9}$, \\ Antonio Cascio ${ }^{9,10}{ }^{(D)}$, Giovanni Mazzola ${ }^{11}$ and Giovanni Guaraldi ${ }^{4,5}$
}

\section{check for}

updates

Citation: Sebastiani, G.; Milic, J.;

Cervo, A.; Saeed, S.; Krahn, T.;

Kablawi, D.; Al Hinai, A.S.;

Lebouché, B.; Wong, P.; Deschenes,

M.; et al. Two-Tier Care Pathways for

Liver Fibrosis Associated to

Non-Alcoholic Fatty Liver Disease in HIV Mono-Infected Patients. J. Pers. Med. 2022, 12, 282. https://doi.org/ 10.3390/jpm12020282

Academic Editor: Lorenza Rimassa

Received: 10 January 2022

Accepted: 10 February 2022

Published: 15 February 2022

Publisher's Note: MDPI stays neutral with regard to jurisdictional claims in published maps and institutional affiliations.

Copyright: (c) 2022 by the authors. Licensee MDPI, Basel, Switzerland. This article is an open access article distributed under the terms and conditions of the Creative Commons Attribution (CC BY) license (https:// creativecommons.org/licenses/by/ $4.0 /)$.
1 Chronic Viral Illness Service, McGill University Health Centre, Montréal, QC H4A 3J1, Canada; adriana.cervo@gmail.com (A.C.); thomas.krahn@mail.mcgill.ca (T.K.); bertrand.lebouche@mcgill.ca (B.L.)

2 Division of Gastroenterology and Hepatology, Royal Victoria Hospital, McGill University Health Centre, Montréal, QC H4A 3J1, Canada; dana.kablawi@mail.mcgill.ca (D.K.); pw0867@gmail.com (P.W.); marc.deschenes@muhc.mcgill.ca (M.D.)

3 Centre for Outcomes Research and Evaluation, Research Institute of the McGill University Health Centre, Montréal, QC H4A 3J1, Canada

4 Department of Surgical, Medical, Dental and Morphological Sciences, University of Modena and Reggio Emilia, 41121 Modena, Italy; jovana.milic@gmail.com (J.M.); giovanni.guaraldi@unimore.it (G.G.)

5 Department of Infectious Diseases, Azienda Ospedaliero-Universitaria di Modena, 41124 Modena, Italy

6 Department of Internal Medicine-Infectious Disease, Center for Dissemination and Implementation, Institute for Public Health, School of Medicine, Washington University, St. Louis, MO 63110-1010, USA; zssaeed@gmail.com

7 Division of Experimental Medicine, McGill University, Montréal, QC H4A 3J1, Canada; al.alhinai@mail.mcgill.ca

8 Department of Family Medicine, McGill University, Montréal, QC H3S 1Z1, Canada

9 Infectious and Tropical Disease Unit, AOU Policlinico "P. Giaccone", 90127 Palermo, Italy; claudiagioe86@gmail.com (C.G.); antonio.cascio03@unipa.it (A.C.)

10 Department of Health Promotion, Mother and Child Care, Internal Medicine and Medical Specialties-University of Palermo, 90127 Palermo, Italy

11 Infectious Diseases Unit, S. Elia Hospital, 93100 Caltanissetta, Italy; gnni.mazzola@gmail.com

* Correspondence: giada.sebastiani@mcgill.ca; Tel.: +39-514-843-1616

Abstract: (1) Background: Developing strategies to identify significant liver fibrosis in people with HIV (PWH) is crucial to prevent complications of non-alcoholic fatty liver disease (NAFLD). We aim to investigate if five simple serum biomarkers applied to $\mathrm{PWH}$ can optimize a care pathway to identify significant liver fibrosis defined by transient elastography (TE). (2) Methods: A two-tier fibrosis pathway was applied to three prospective cohorts of PWH undergoing TE with CAP. NAFLD was diagnosed as a controlled attenuation parameter $\geq 248 \mathrm{~dB} / \mathrm{m}$. Five simple fibrosis biomarkers (FIB-4 < 1.3, BARD score 0-1, NAFLD fibrosis score $<-1.455$, AST:ALT ratio $<0.8$ and APRI $<0.5$ ) were applied as first-tiers to exclude significant liver fibrosis. We determined the decrease in referral for TE that would have occurred based on biomarker assessment and the discordance between low simple fibrosis biomarkers and high TE ( $\geq 7.1 \mathrm{kPa})$, indicating significant liver fibrosis. (3) Results: Of the 1749 consecutive PWH, 15.1\% had significant liver fibrosis by TE and 39.1\% had NAFLD. The application of the fibrosis biomarkers as first tiers would have resulted in a decrease in TE referrals between $24.9 \%$ (BARD score) and $86.3 \%$ (APRI). The lowest discordance rate was with NAFLD fibrosis score (8.5\%). After adjustments, BMI (odds ratio (OR) 1.12, 95\% CI: 1.08-1.17) and triglycerides (OR 1.26, 95\% CI: 1.11-1.44) were independent predictors of discordance for APRI $<0.5$ and TE $\geq 7.1$. The performance of the two-tier pathways was similar in PWH with and without NAFLD. (4) Conclusions: Implementing a two-tier pathway could save a substantial proportion up of TE examinations, reducing costs and helping resource optimization in HIV care. Patients with metabolic risk factors for NAFLD and low fibrosis biomarker may still be considered for TE referral. 
Keywords: transient elastography; controlled attenuation parameter; serum fibrosis biomarkers; APRI; FIB-4

\section{Introduction}

Non-alcoholic fatty liver disease (NAFLD) represents a global epidemic, with a prevalence at $25.24 \%$ [1]. If left unmanaged, NAFLD could lead to non-alcoholic steatohepatitis (NASH), liver fibrosis accumulation, cirrhosis and end-stage liver complications. NAFLD seems frequent in people with HIV (PWH), with a prevalence ranging from 13 to 65\% [2-7]. This burden is likely driven by a multifactorial complex pathogenesis, resulting from lifelong use of ART, past exposure to hepatotoxic d-drugs (didanosine and stavudine), persistent immune activation and HIV-related inflammation, and very prevalent dysmetabolic conditions [8,9]. NAFLD may not only be more prevalent in PWH than in the HIV-uninfected population, but also more severe: both NASH and significant liver fibrosis seem at least twice more frequent in HIV mono-infected patients than in the general population [10-14].

NAFLD is often asymptomatic until patients develop hepatic decompensation, with significant morbidity and mortality and related socio-economic burden [15]. The stage of liver fibrosis represents the main prognostic factor of all-cause and liver-related mortality in NAFLD [16]. There is a need for personalized medicine and implementation of strategies to identify those who have significant liver fibrosis and are at risk of poor outcomes. The guidelines from the European AIDS Clinical Society (EACS) recommend the case-finding of significant liver fibrosis in $\mathrm{PWH}$ with metabolic conditions or persistent elevated transaminases [17]. These recommendations are in line with other at-risk populations for NAFLD-related liver fibrosis, such as patients with type-2 diabetes $[18,19]$. Transient elastography (TE) with controlled attenuation parameter (CAP) is a feasible and accurate tool to assess both hepatic steatosis and NAFLD-associated liver fibrosis in PWH [20]. The utilization of this specialist test facilitates the implementation of therapeutic interventions and surveillance for complications associated with advanced liver fibrosis, including screening for hepatocellular carcinoma (HCC) and esophageal varices. However, diagnosing liver fibrosis is a challenge due to the large number of PWH at risk for NAFLD, as well as the additional resources of delivering TE with CAP, which is often not readily accessible in clinics practicing HIV care. Clinical pathways have been proposed in HIV-uninfected NAFLD to screen for liver fibrosis in at-risk populations and reduce the need for specialist tests [21-23]. In these models, readily available and inexpensive fibrosis biomarkers with high negative predictive value (NPV) are used as first-tier tests, while more specialized tests, such as TE with CAP, are second-tier tests reserved to cases in which fibrosis cannot be excluded by the simple biomarker [21,23]. Among the simple fibrosis biomarkers potentially suitable as first-tier tests, the aspartate aminotransferase (AST): alanine aminotransferase (ALT) ratio, the fibrosis-4 (FIB-4), the AST-to-Platelet Ratio Index (APRI), the NAFLD fibrosis score and the BARD score have been sufficiently validated for the diagnosis of significant liver fibrosis, as well as for their prognostic value in predicting liver-related events and mortality [24,25]. The performance characteristics of the clinical pathways employing simple fibrosis biomarkers and TE with CAP for significant liver fibrosis in PWH, who are at risk for NAFLD, are unknown.

The aim of this study is to investigate if five simple serum biomarkers applied to $\mathrm{PWH}$ can optimize a two-tier care pathway for the identification of significant liver fibrosis as defined by TE. Specifically, we evaluate the reduction in the TE referral rate and related costs that would have occurred based on biomarker assessment, and the discordance rate between TE and simple fibrosis biomarkers. Finally, we determine the factors associated with the discordance between serum biomarkers and TE. 


\section{Materials and Methods}

We conducted a retrospective cross-sectional study from the LIVEr disease in HIV (LIVEHIV), Modena HIV Metabolic Clinic (MHMC) and Liver pathologies in HIV in Palermo (LHIVPA) Cohorts $[10,26,27]$. The LIVEHIV Cohort is a prospective routine screening program for NAFLD and liver fibrosis established in September 2013 at McGill University Health Centre (MUHC) in Montreal, Canada. HIV-infected patients undergo screening for NAFLD and liver fibrosis by TE with CAP [10]. The MHMC Cohort was initiated in 2004 in Modena, Italy, to assess longitudinal metabolic changes among PWH through annual comprehensive assessment and TE with CAP [27]. The LHIVPA Cohort was initiated in 2011 at the Infectious Diseases Outpatient Clinic of the University Hospital in Palermo, Italy. Metabolic assessment through physical and biochemical parameters and TE with CAP is conducted annually [28]. We included all consecutive patients with HIV infection (documented by positive enzyme-linked immunosorbent assay (ELISA) with Western blot confirmation) aged $\geq 18$ years with availability of TE with CAP and relevant clinical and biochemical parameters. Exclusion criteria were: (i) positivity for $\mathrm{HCV}$ antibody or hepatitis B surface antigen; (ii) evidence of other liver disease; (iii) significant alcohol intake, defined as more than $30 \mathrm{~g} /$ day in men and more than $20 \mathrm{~g} /$ day in women [19]; (iv) history of HCC or liver transplantation; (v) contraindications (pregnancy, pacemaker insertion) and failure or unreliable measurement of TE examination with CAP; and (vi) missing liver transaminases or platelets.

We included patients with available data within 3 months from the TE examination, namely demographic information, HIV and medications history, BMI, liver serum biomarkers, lipid profile, hematological and immuno-virological parameters. Type-2 diabetes mellitus was defined as a hemoglobin glycosylated of $6.5 \%$ or greater, or as previously diagnosed by an endocrinologist/treating physician. Hypertriglyceridemia was defined as triglycerides $\geq 1.7 \mathrm{mmol} / \mathrm{L}$. Elevated liver transaminases were defined as either ALT $>45 \mathrm{IU} / \mathrm{L}$ or AST > $35 \mathrm{IU} / \mathrm{L}$.

TE examinations were performed on a $4 \mathrm{~h}$ fasting patient by a maximum of two experienced operators at each site ( $>500$ examinations before the study) [29]. The standard $\mathrm{M}$ probe was used in all patients. The XL probe was used in the case of failure with $\mathrm{M}$ probe and if $\mathrm{BMI}>30 \mathrm{Kg} / \mathrm{m}^{2}$ [30]. The following criteria were applied to define the result of liver stiffness measurement (LSM) as reliable: at least 10 validated measures and an interquartile range $<30 \%$ of the median. A cut-off of LSM by TE $\geq 7.1 \mathrm{kPa}$ was used to define significant liver fibrosis, corresponding to stage F2-F4 out of 4 by the histologic Kleiner staging system [31,32]. NAFLD was defined as CAP $\geq 248 \mathrm{~dB} / \mathrm{m}[7,33]$.

Five simple fibrosis biomarkers were adopted as first-tier tests. Previously proposed validated cut-offs were used to rule-out significant liver fibrosis and subsequent need for the second-tier test TE. The five biomarkers included:

- $\quad$ AST:ALT ratio [34]. A cut-off < 0.8 was used to exclude significant liver fibrosis $[24,35]$.

- $\quad$ AST-to-Platelet Ratio Index $($ APRI $)=($ AST $/$ upper limit of normal $) /$ platelets $\times 100$. A cut-off $<0.5$ was used to exclude significant liver fibrosis [36].

- $\quad$ FIB-4 $=($ age $\times$ AST $) /$ platelets $\times \sqrt{ }$ ALT. A cut-off $<1.30$ was used to exclude significant liver fibrosis $[24,37]$.

- $\quad$ BARD $=$ BMI $\geq 28 \mathrm{~kg} / \mathrm{m}^{2}($ yes $=1$, no $=0)+$ AST:ALT ratio $\geq 0.8($ yes $=2$, no $=0)+$ type-2 diabetes (yes $=1$, no $=0$ ). A cut-off $<2$ was used to exclude significant liver fibrosis [38].

- $\quad$ NAFLD fibrosis score $=-1.675+(0.037 \times$ age $)+(0.094 \times$ BMI $)+(1.13 \times$ diabetes $($ yes $=1$, no $=0))+(0.99 \times$ AST:ALT ratio $)-(0.013 \times$ platelets $)-(0.66 \times$ albumin $(\mathrm{g} / \mathrm{dL}))$. A cut off $<-1.455$ was used to exclude significant liver fibrosis [39].

The primary study outcome was the decrease in TE referral rate that would have occurred when using simple fibrosis biomarkers as first-tier tests in a two-tier pathway (Figure 1A), compared to the use of TE in all patients (Figure 1B). Secondary outcomes include: (a) rate of discordance between high TE ( $\geq 7.1 \mathrm{kPa}$ ) and each among low simple fibrosis biomarker (FIB-4 < 1.3, BARD score 0-1, NAFLD fibrosis score $<-1.455$, AST:ALT 
ratio $<0.8$ and APRI $<0.5)$; (b) predictors of discordance between high TE $(\geq 7.1 \mathrm{kPa})$ and low simple fibrosis biomarkers; (c) cost analysis of two-tier pathways compared to the use of TE in all patients.

A)

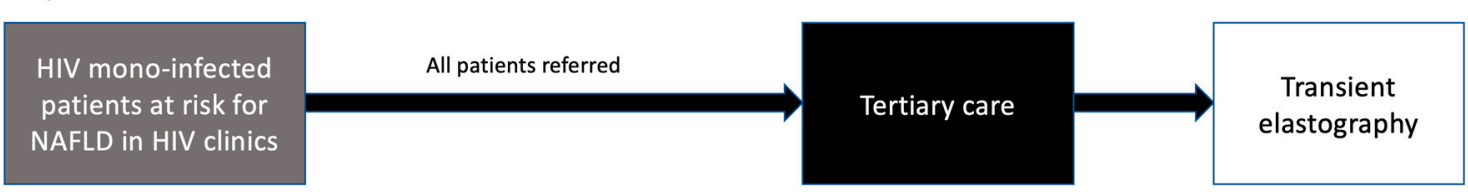

B)

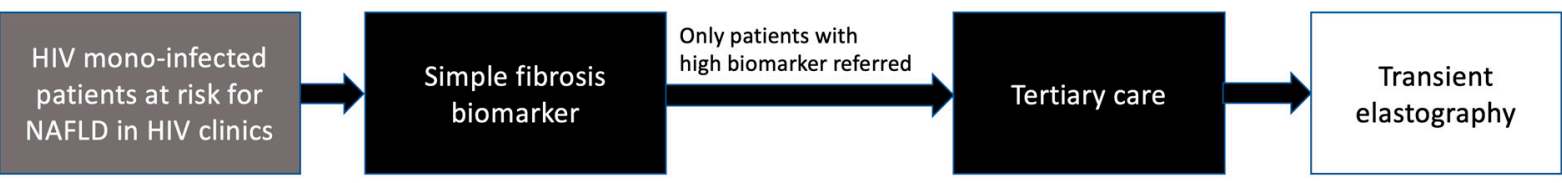

Figure 1. Schematic representation of the two scenarios of referral to tertiary care. The first scenario (A) assumes that patients have simple a fibrosis biomarker tested in primary care and those at risk for significant liver fibrosis are referred to tertiary care to undergo transient elastography. The second scenario (B) assumes that all patients are referred to tertiary care to undergo transient elastography. High simple fibrosis biomarker was defined as follows: FIB-4 $\geq 1.3$, BARD score 2-4, NAFLD fibrosis score $\geq-1.455$, AST:ALT ratio $\geq 0.8$ or APRI $\geq 0.5$.

Potential direct cost savings were estimated using Canadian data. As previously reported $[40,41]$, costs of non-invasive tests in Canadian dollars were as follows: FIB-4 and APRI: CAD 17; NAFLD fibrosis score: CAD 22; AST:ALT ratio and BARD score: CAD 10; TE: CAD 125. Total direct cost saved was computed by subtracting the direct cost of serum biomarker to the cost saved for the TE examination.

We compared the characteristics of the participants by significant liver fibrosis status by TE using Student's t-test for continuous variables and Pearson's $\chi^{2}$ for categorical variables. The two-tier pathways were modelled using one of the simple fibrosis biomarkers as firsttier and TE as the second-tier test. The reduction in the referral rate for TE tests, along with the discordance rate, was computed. A subgroup analysis was conducted in patients with NAFLD diagnosed by CAP. Predictors of discordance between low simple fibrosis biomarkers and TE $\geq 7.1 \mathrm{kPa}$ were determined using unadjusted and adjusted logistic regression models and reported as adjusted odds ratios (aOR) with 95\% confidence interval (CI). All adjusted regression models included covariates that were determined a priori to be clinically important, based on the previous literature, or those with $p$-value $<0.05$ in univariable analysis. Final models were adjusted for age, sex, BMI, diabetes, triglycerides, and CD4 cell count. A complete case analysis was used for the multivariable models and the percentage of missing data were less than $15 \%$, unless otherwise indicated. All tests were two-tailed and with a significance level of $\alpha=0.05$. Statistical analyses were performed using STATA 15 (STATA Corp. LP, College Station, TX, USA).

\section{Results}

After applying the inclusion and exclusion criteria, a total of $1749 \mathrm{PWH}$ were included (Figure 2), of whom 582 were from the LIVEHIV cohort, 649 from the MHMC cohort and 518 from the LHIVPA cohort. 


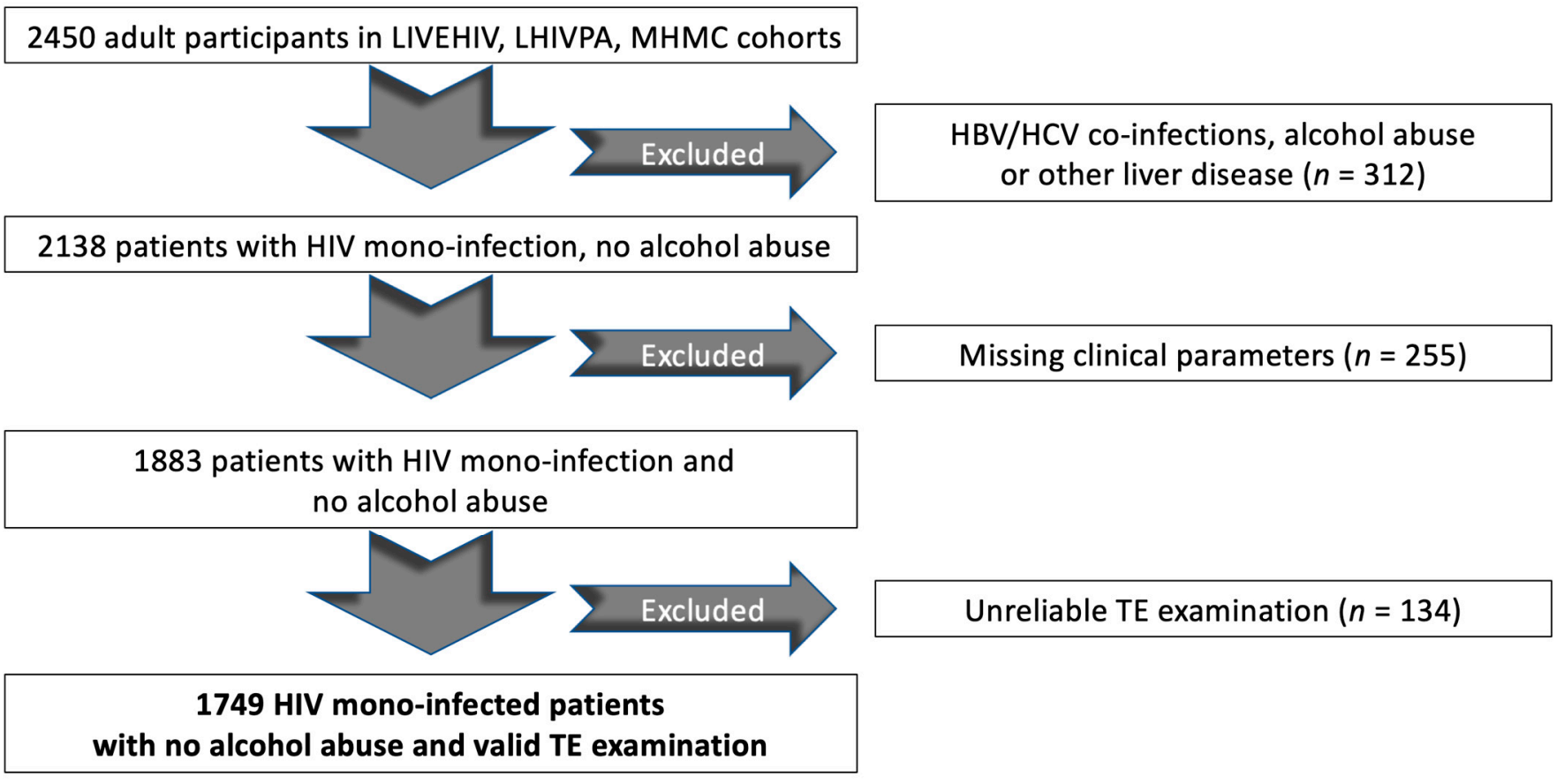

Figure 2. Flow chart displaying the selection of study participants in the cohort. Abbreviations: LIVEHIV, LIVEr disease in HIV; MHMC, Modena HIV Metabolic Clinic; LHIVPA, Liver pathologies and HIV in Palermo; HBV, hepatitis B virus; HCV, hepatitis C virus; HIV, human immunodeficiency virus; TE, transient elastography.

Mean age was 50.2 years (standard deviation (SD) 10.4) and $74.5 \%$ were males. A total of $1483(84.8 \%)$ PWH included were of white ethnicity. The mean CD4 cell count was 702.9 cells $/ \mu \mathrm{L}$ (SD 318.3) and mean duration of HIV infection was 15.5 years (SD 9.8). Most of the PWH included in this study had well controlled HIV: the proportion of participants with a CD4 cell count $<200$ cells $/ \mathrm{mm}^{3}$ and $<350$ cells $/ \mathrm{mm}^{3}$ was $2.6 \%$ and $11.3 \%$, respectively. All patients were on ART, including 1497 (85.6\%) on nucleoside/nucleotide reverse transcriptase inhibitors, 773 (44.2\%) on nonnucleoside reverse transcriptase inhibitors, 932 $(53.3 \%)$ on protease inhibitors and $619(35.4 \%)$ on integrase inhibitors. Overall, 132 (7.5\%) had a history of exposure to didanosine. The distribution of risk factors for NAFLD was as follows: $44.8 \%$ PWH were overweight, 34\% had diabetes and $12.8 \%$ had elevated liver transaminases. In the whole cohort, 264 (15.1\%) had significant liver fibrosis by LSM. Table 1 reports the characteristics of the study population with univariable analysis by significant liver fibrosis status, as per LSM. When compared to patients without significant liver fibrosis, PWH with fibrosis were older, had higher BMI and longer time since HIV diagnosis. They also had a higher prevalence of diabetes. Finally, PWH with significant liver fibrosis had higher ALT, AST, total cholesterol, triglycerides, albumin, CAP, while having lower platelets. They also presented a tendency for lower CD4 cell count. All the serum fibrosis biomarkers were higher in PWH with significant liver fibrosis by LSM, except for the AST:ALT ratio. 
Table 1. Characteristics of PWH by significant liver fibrosis status as determined by LSM with transient elastography $(n=1749)$.

\begin{tabular}{|c|c|c|c|}
\hline & $\begin{aligned} \mathrm{LSM} & \geq 7.1 \mathrm{kPa} \\
(n=264) & \end{aligned}$ & $\begin{array}{c}\text { LSM }<7.1 \mathrm{kPa} \\
\quad(n=1485)\end{array}$ & $p$ \\
\hline Age (years) & $53.1(9.4)$ & $49.7(10.5)$ & $<0.001$ \\
\hline Male sex (\%) & $203(76.9)$ & $1100(74.1)$ & 0.333 \\
\hline \multicolumn{4}{|l|}{ Ethnicity $(\%)$} \\
\hline White/Caucasian & $231(87.5)$ & $1250(84.2)$ & \\
\hline Black non-Hispanic & $25(9.5)$ & $168(11.3)$ & 0.167 \\
\hline Diabetes $(\%)$ & $110(41.7)$ & $484(32.6)$ & $<0.001$ \\
\hline \multirow{2}{*}{$\begin{array}{c}\text { BMI }\left(\mathrm{Kg} / \mathrm{m}^{2}\right)^{\circ} \\
\text { Time since HIV diagnosis } \\
\text { (vears) }\end{array}$} & $27.2(5.3)$ & $24.7(4.0)$ & $<0.001$ \\
\hline & $18.9(10.3)$ & $14.9(9.6)$ & $<0.001$ \\
\hline $\begin{array}{c}\text { Undetectable HIV viral load } \\
(<40 \text { copies } / \mathrm{mL})(\%)\end{array}$ & $203(77.0)$ & $1099(74.0)$ & 0.322 \\
\hline CD4 cell count (cells $/ \mu \mathrm{L})$ & $669.3(347.4)$ & $708.9(312.6)$ & 0.065 \\
\hline \multicolumn{4}{|l|}{ Current ART regimen $(\%)$} \\
\hline NRTIs & $228(86.4)$ & $1268(85.4)$ & 0.678 \\
\hline NNRTIs & $122(46.2)$ & 649 (43.7) & 0.449 \\
\hline Protease inhibitors & $148(56.1)$ & 768 (51.7) & 0.192 \\
\hline Integrase inhibitors & $83(31.4)$ & $538(36.2)$ & 0.134 \\
\hline $\begin{array}{c}\text { Past exposure to didanosine } \\
(\%)\end{array}$ & $23(8.7)$ & $107(7.2)$ & 0.390 \\
\hline $\operatorname{ALT}(\mathrm{IU} / \mathrm{L})$ & $35.5(32.3)$ & $24.5(16.1)$ & $<0.001$ \\
\hline AST (IU/L) & $31.7(24.2)$ & $23.1(10.4)$ & $<0.001$ \\
\hline $\begin{array}{c}\text { Platelets } \\
\left(10^{9} \text { cells } / \mathrm{L}\right)\end{array}$ & $202.3(74.3)$ & $223.8(62.3)$ & $<0.001$ \\
\hline Albumin (g/dL) & $4.32(0.55)$ & $4.37(0.40)$ & 0.119 \\
\hline Triglycerides (mmol/L) & $1.96(1.76)$ & $1.51(1.06)$ & $<0.001$ \\
\hline Total cholesterol (mmol/L) & $3.58(1.89)$ & $2.97(2.23)$ & $<0.001$ \\
\hline $\mathrm{HDL}(\mathrm{mmol} / \mathrm{L})$ & $1.18(0.38)$ & $1.28(0.39)$ & $<0.001$ \\
\hline $\mathrm{CAP}(\mathrm{dB} / \mathrm{m})$ & $269.2(61.7)$ & $230.8(54.2)$ & $<0.001$ \\
\hline AST: ALT ratio & $1.07(0.65)$ & $1.09(0.44)$ & 0.375 \\
\hline BARD score & $2.20(1.14)$ & $2.01(1.11)$ & 0.015 \\
\hline NAFLD fibrosis score & $-0.84(1.55)$ & $-1.70(1.36)$ & $<0.001$ \\
\hline FIB- 4 & $1.91(2.00)$ & $1.20(0.67)$ & $<0.001$ \\
\hline APRI & $0.60(0.82)$ & $0.33(0.20)$ & $<0.001$ \\
\hline
\end{tabular}

Notes: Continuous variables are expressed as mean (standard deviation) and categorical variables as number (\%). The $p$-values refer to Student $t$-test or $\chi^{2}$ test between LSM $\geq 7.1$ and LSM $<7.1 \mathrm{kPa}$. Data on BMI and BARD score were available for $1564 \mathrm{PWH}(89.4 \%)$. Data on albumin were available for $1122 \mathrm{PWH}(64.2 \%)$. Data on NAFLD fibrosis score were available for $1017 \mathrm{PWH}(58.1 \%)$.

\subsection{Application of the Two-Tier Pathway}

The application of the simple fibrosis biomarkers as first-tier tests would have resulted in a significant proportion of PWH identified as not at risk for significant liver fibrosis, with consequent decrease in TE referrals ranging between 24.9\% (BARD score) and $86.3 \%$ (APRI) (Table 2). The discordance rate between low fibrosis biomarker and high LSM ranged between $8.5 \%$ (NAFLD fibrosis score) and 19.5\% (AST: ALT ratio).

Table 2. Cost analysis of potential direct cost savings by the two-tier pathway, estimated using Canadian data.

\begin{tabular}{cccccc}
\hline & APRI & FIB-4 & $\begin{array}{c}\text { NAFLD Fibrosis } \\
\text { Score }\end{array}$ & $\begin{array}{c}\text { BARD } \\
\text { Score }\end{array}$ & $\begin{array}{c}\text { AST: ALT } \\
\text { Ratio }\end{array}$ \\
\hline $\begin{array}{c}\text { Decrease in TE referral (\%) } \\
\text { Discordance high LSM/ } \\
\text { low biomarker (\%) }\end{array}$ & 86.3 & 63.0 & 51.2 & 24.9 & 26.2 \\
$\begin{array}{c}\text { Direct cost of serum } \\
\text { biomarker per 100 PWH } \\
\text { (CAD) }\end{array}$ & 11.7 & 11.0 & 8.5 & 15.4 & 19.5 \\
$\begin{array}{c}\text { TE cost saved per 100 PWH } \\
\quad \text { (CAD) }\end{array}$ & 1700 & 1700 & 2200 & 1000 & 1000 \\
$\begin{array}{c}\text { Total direct cost saved per } \\
\text { 100 PWH (CAD) }\end{array}$ & 9088 & 6175 & 4200 & 3113 & 3275 \\
\hline
\end{tabular}

Notes: All dollar values are 2019 Canadian dollars. 
Since APRI and FIB-4 yielded the highest decrease in TE referrals, we conducted a multivariable analysis of independent predictors of discordance between low APRI $(<0.5)$ or FIB-4 $(<1.3)$ and high TE $(\geq 7.1)$. After adjustments, high BMI and triglycerides were independent predictors of discordance for APRI and FIB-4 (Table 3).

Table 3. Multivariable analysis of predictors of discordance between high TE and low FIB-4 $(n=1101)$ or low APRI $(n=1510)$.

\begin{tabular}{cccc}
\hline Variable & OR $(\mathbf{9 5} \% \mathbf{C I})$ & aOR $(\mathbf{9 5} \% \mathbf{C I})$ & $p$-Value \\
\hline & FIB-4 & & \\
Male sex (yes vs. no) & $0.91(0.60-1.39)$ & $0.82(0.51-1.31)$ & 0.052 \\
BMI (per Kg/m $\left.{ }^{2}\right)$ & $1.14(1.09-1.19)$ & $1.14(1.09-1.19)$ & $<0.001$ \\
Diabetes (yes vs. no) & $0.83(0.56-1.28)$ & $0.77(0.48-1.23)$ & 0.270 \\
Triglycerides (per mmol/L) & $1.29(1.11-1.49)$ & $1.23(1.03-1.45)$ & 0.019 \\
CD4 cell count (per 100 cell/mL) & $0.99(0.99-1.00)$ & $0.99(0.99-1.00)$ & 0.807 \\
& APRI & & \\
Age (per 10 years) & $1.27(1.09-1.48)$ & $1.19(0.99-1.42)$ & 0.054 \\
Male sex (yes vs. no) & $0.95(0.67-1.35)$ & $0.74(0.50-1.09)$ & 0.129 \\
BMI (per Kg/m 2 ) & $1.12(1.08-1.16)$ & $1.12(1.08-1.17)$ & $<0.001$ \\
Diabetes (yes vs. no) & $1.21(0.87-1.68)$ & $1.13(0.78-1.64)$ & 0.511 \\
Triglycerides (per mmol/L) & $1.32(1.17-1.48)$ & $1.26(1.11-1.44)$ & 0.001 \\
CD4 cell count (per 100 cell/mL) & $0.99(0.99-1.00)$ & $0.99(0.99-1.00)$ & 0.154
\end{tabular}

Notes: Odds ratios (OR) and 95\% confidence interval (CI) are presented for each variable in the unadjusted and adjusted analysis. Continuous variables in the models were age, BMI, triglycerides, and CD4 cell count. Categorical variables in the models were male sex and diabetes.

In the cost analysis, APRI showed the highest total direct cost saved (CAD 9088 per 100 PWH), followed by FIB-4 (CAD 6175 per 100 PWH), NAFLD fibrosis score, AST:ALT ratio and BARD score (Table 2 ).

\subsection{Effect of NAFLD}

The overall prevalence of NAFLD in our cohort of consecutive PWH was 39.1\%. The prevalence of significant liver fibrosis by LSM was higher in patients with NAFLD (24.3\%) compared to those without NAFLD (9.2\%) $(p<0.001)$. Patients with NAFLD had higher rates of discordance low fibrosis biomarker and high LSM by TE (Figure 3). Conversely, there was no difference in decrease in TE referral according to NAFLD status (data not shown).

Table 4 shows the characteristics of the 264 PWH with significant liver fibrosis by NAFLD status. Those PWH with significant fibrosis but without NAFLD had lower BMI, CD4 cell count, platelets, total cholesterol and triglycerides. They also had higher HDL cholesterol, AST: ALT ratio, FIB-4 and APRI. Finally, PWH with significant liver fibrosis without NAFLD was in a higher proportion in females and in the parameter of exposure to didanosine. 


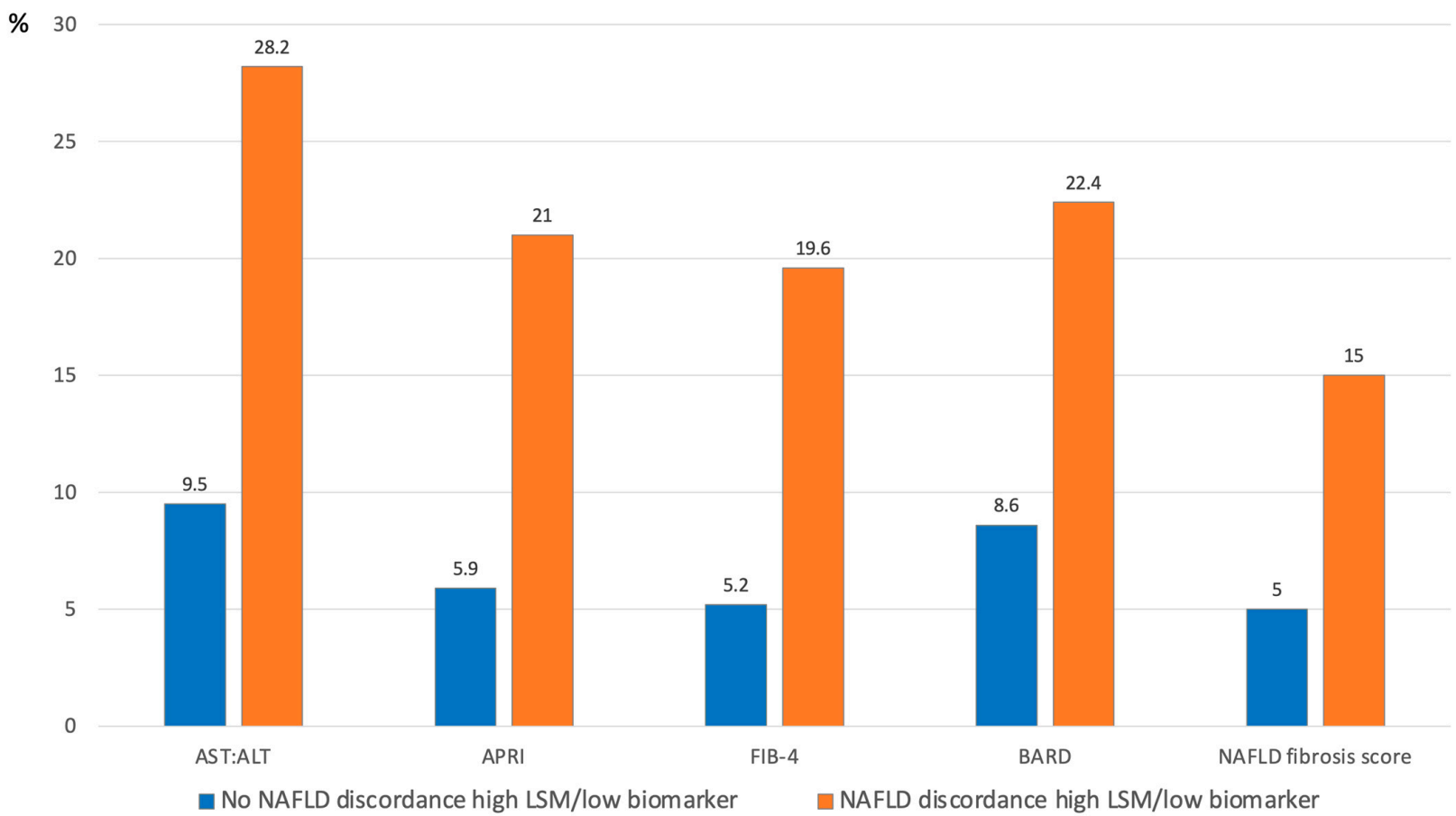

Figure 3. Proportion of discordance between low fibrosis biomarker (first-tier test) and high LSM by TE (second-tier test) by NAFLD status.

Table 4. Characteristics of PWH with significant liver fibrosis by transient elastography by NAFLD status $(n=264)$.

\begin{tabular}{|c|c|c|c|}
\hline & $\begin{array}{c}\mathrm{CAP} \geq 248 \mathrm{~dB} / \mathrm{m} \\
(\mathrm{n}=166)\end{array}$ & $\begin{array}{c}\mathrm{CAP}<248 \mathrm{~dB} / \mathrm{m} \\
(\mathrm{n}=98)\end{array}$ & $p$-Value \\
\hline Age (years) & $53.6(8.9)$ & $52.3(10.2)$ & 0.293 \\
\hline Male sex $(\%)$ & $135(81.3)$ & $68(69.4)$ & 0.026 \\
\hline \multicolumn{4}{|l|}{ Ethnicity $(\%)$} \\
\hline White/Caucasian & $139(83.7)$ & $92(93.9)$ & \\
\hline Black non-Hispanic & $17(10.2)$ & $8(8.2)$ & 0.581 \\
\hline Diabetes $(\%)$ & $66(60.0)$ & $44(40.0)$ & 0.413 \\
\hline $\operatorname{BMI}\left(\mathrm{Kg} / \mathrm{m}^{2}\right)^{\circ}$ & $28.7(4.7)$ & $24.4(5.2)$ & $<0.001$ \\
\hline Time since HIV diagnosis (years) & $18.5(9.7)$ & $19.5(11.3)$ & 0.435 \\
\hline $\begin{array}{l}\text { Undetectable HIV viral load } \\
(<40 \text { copies } / \mathrm{mL})(\%)\end{array}$ & $125(75.3)$ & $78(79.6)$ & 0.639 \\
\hline CD4 cell count (cells $/ \mu \mathrm{L})$ & $711.3(656.8)$ & $599.3(330.7)$ & 0.012 \\
\hline \multicolumn{4}{|l|}{ Current ART regimen $(\%)$} \\
\hline NRTIs & $141(84.9)$ & $87(88.8)$ & 0.380 \\
\hline NNRTIs & $80(48.3)$ & $42(43.9)$ & 0.401 \\
\hline Protease inhibitors & $101(60.8)$ & $62(63.3)$ & 0.696 \\
\hline Integrase inhibitors & $48(28.9)$ & $35(35.7)$ & 0.250 \\
\hline Past exposure to didanosine (\%) & $6(3.6)$ & 17 (17.3) & $<0.001$ \\
\hline ALT (IU/L) & $38.0(34.9)$ & $31.3(27.0)$ & 0.105 \\
\hline AST (IU/L) & 30.7 (23.7) & $33.3(24.9)$ & 0.410 \\
\hline Platelets $\left(10^{9}\right.$ cells $\left./ \mathrm{L}\right)$ & $214.2(68.9)$ & $182.1(78.9)$ & $<0.001$ \\
\hline Albumin $(\mathrm{g} / \mathrm{L})$ & $43.5(5.4)$ & $42.6(5.7)$ & 0.233 \\
\hline Triglycerides $(\mathrm{mmol} / \mathrm{L})$ & $2.3(2.1)$ & $1.4(0.8)$ & $<0.001$ \\
\hline Total cholesterol (mmol/L) & $4.6(1.1)$ & 1.9 (1.7) & $<0.001$ \\
\hline $\mathrm{HDL}(\mathrm{mmol} / \mathrm{L})$ & $1.1(0.4)$ & $1.3(0.4)$ & 0.012 \\
\hline AST:ALT ratio & $0.93(0.34)$ & $1.29(0.93)$ & $<0.001$ \\
\hline BARD score & $2.19(1.14)$ & $2.22(1.14)$ & 0.818 \\
\hline NAFLD fibrosis score & $-0.95(1.41)$ & $-0.66(1.75)$ & 0.247 \\
\hline FIB-4 & 1.50 (1.11) & $2.61(2.83)$ & $<0.001$ \\
\hline APRI & $0.50(0.61)$ & 0.77 (1.07) & 0.008 \\
\hline
\end{tabular}

Notes: Continuous variables are expressed as mean (standard deviation) and categorical variables as number (\%) The $p$-values refer to Student $t$-test or $\chi^{2}$ test between CAP $\geq 248 \mathrm{~dB} / \mathrm{m}$ and CAP $<248 \mathrm{~dB} / \mathrm{m}$. ${ }^{\circ} \mathrm{Data}$ on $\mathrm{BMI}$ and BARD score were available for $237 \mathrm{PWH}(89.8 \%)$. Data on albumin were available for $183 \mathrm{PWH}(69.3 \%)$. Data on NAFLD fibrosis score were available for $159 \mathrm{PWH}(60.2 \%)$. 


\section{Discussion}

A two-tier care pathway using simple fibrosis biomarkers as first-tier tests in a large population of consecutive $\mathrm{PWH}$ could reduce the referral rate to tertiary care for TE examination up to $86 \%$, with a significant reduction in terms of cost and use of healthcare resources. We also found that APRI and FIB-4 reduced the most referral rates and costs, while the NAFLD fibrosis score had the lowest discordance rate with LSM. Finally, our study indicated that discordance rates between FIB-4 or APRI and LSM are associated with overweight and hypertriglyceridemia, as such TE examination may still be considered in $\mathrm{PWH}$ even in the case of low fibrosis biomarker.

With the increasing life expectancy of PWH thanks to the widespread use of ART, NAFLD is now a major contributor to the burden of liver disease. Studies across different countries place the prevalence of the disease between 28 and 72.1\% [20]. NAFLD seems not only more frequent in the setting of HIV infection, but also more severe. NASH has been reported in up to $63.6 \%$ of HIV mono-infected patients with chronically elevated ALT and in $10 \%$ of those attending a routine screening program [13,20,42-44]. PWH with NAFLD have more advanced liver disease and higher rates of NASH compared to age/sexmatched HIV-negative controls [11]. The prevalence of NAFLD-associated significant liver fibrosis ranges between 13.8 and $28.6 \%$ [20,43,45]. Similarly, in our study we found a prevalence of significant liver fibrosis of $15.1 \%$. Unfortunately, NAFLD lacks specific signs or symptoms, and between $67 \%$ and $83 \%$ of PWH with NAFLD have normal ALT [7,46]. As such, a significant proportion of NAFLD patients receive a diagnosis at the first episode of hepatic decompensation due to liver cirrhosis [47]. Similarly to other at-risk populations for NAFLD, such as patients with type-2 diabetes [18,19], recent EACS guidelines recommend the staging of liver fibrosis in PWH at risk for NAFLD, particularly in case of metabolic comorbidities [17]. Due to the high prevalence of NAFLD in PWH, it is unfeasible to perform tertiary care specialized tests for fibrosis staging in all patients, such as TE. Twotier care pathways have been recently proposed in populations at risk for NAFLD, such as patients with type-2 diabetes or those with unexplained elevation of liver transaminases. In primary care, these pathways involve the use of simple fibrosis biomarkers to stratify patients at risk for NAFLD who have a higher likelihood of having significant liver fibrosis. These readily available simple fibrosis biomarkers used as first-tier tests have low cost and high NPV. Davyduke et al. reported that a FIB-4 first care pathway used in 565 patients at risk for NAFLD from primary care could save $87 \%$ of specialist tests, including TE and liver biopsy [21]. Similarly, a U.K. study of 3012 patients with risk factors for NAFLD found that a pathway using FIB-4 followed by more specialized fibrosis tests could save $88 \%$ unnecessary referrals to tertiary care [23]. With up to $86 \%$ saved TE examinations, our study achieved similar results in the specific setting of HIV mono-infection. Indeed, biopsy-proven studies in PWH showed a similarly high NPV of simple fibrosis biomarkers to exclude significant liver fibrosis. In $49 \mathrm{PWH}$, Lemoine et al. reported a NPV of $95 \%$ for FIB- $4<1.30,92 \%$ for APRI $<0.5$ and $90 \%$ for NAFLD fibrosis score $<-1.455$ compared to liver histology [31]. In the two-tier pathway we used, only patients with a high fibrosis biomarker would be referred to tertiary care for TE. Our study found that APRI and FIB-4 had the highest rates of decrease in TE referral to tertiary care, $86.3 \%$ and $60 \%$, respectively. These figures would also result in a reduction in costs: compared to the use of TE in all PWH, the APRI first and FIB-4 first pathways would save CAD 9088 and CAD 6175 per $100 \mathrm{PWH}$, respectively. As for NAFLD fibrosis score, although the decrease in TE referrals and costs was inferior, the discordance rate between this biomarker and LSM by TE was the lowest among the simple fibrosis biomarkers investigated. Conversely, our study showed that the simple fibrosis biomarkers AST:ALT ratio and BARD score perform less than APRI, FIB-4 and NAFLD fibrosis score in a two-tier pathway in PWH. These results are in agreement with a report of $743 \mathrm{PWH}$ without viral hepatitis, where TE and simple liver fibrosis scores showed a significant degree of discordance, proposing to limit that use of single diagnostic tools in routine clinical practice [48]. The multivariable analysis found that high BMI and triglycerides were independent predictors associated with a discordance 
between low APRI $(<0.5)$ or FIB-4 $(<1.3)$ and high LSM $(\geq 7.1)$. This finding could be used in clinical practice, suggesting that $\mathrm{PWH}$ with these metabolic comorbidities may be considered for TE referral even in the case of low APRI or FIB-4, which in fact do not contain any metabolic variable in their formula. This was further strengthened by the fact that PWH with NAFLD diagnosed by CAP had higher rates of discordance for the simple fibrosis biomarkers. Giving that both BMI and triglyceride levels are dynamic variables, they should be reassessed at each time point when the two-tier care pathway is applied. This is particularly relevant for BMI, given that recent studies suggest weight gain overtime with specific ART regimens, such as integrase inhibitors [49]. Moreover, dietary patterns, such as the Mediterranean diet, can influence both BMI and triglycerides in NAFLD, thus pointing out the need for the longitudinal re-assessment of these variables [50]. The EACS guidelines recommend periodical re-assessment of fibrosis every $2-5$ years, depending on clinical risk [17].

The overall prevalence of NAFLD in our at-risk cohort was 39.1\%, a figure which is in line with previous reports in PWH cohorts and with a meta-analysis $[4,10,27]$. In PWH with NAFLD, we found a higher prevalence of significant liver fibrosis by TE $(24.3 \%)$ compared to those without NAFLD. However, the prevalence of significant liver fibrosis in PWH without NAFLD was still $9.2 \%$, pointing out that HIV mono-infected patients can develop significant liver fibrosis in the absence of NAFLD. These liver fibrosis cases may be due to risk factors for liver disease other than the metabolic ones and that are unique to HIV infection, including chronic inflammation and exposure to ART [20,51]. Indeed, patients with significant liver fibrosis and without NAFLD had a lower CD4 cell count and a higher proportion of exposure to didanosine, while metabolic comorbidities and high BMI were less frequent. Previous reports suggested low CD4 cell counts to be associated with liver fibrosis [52]. Even in the group of PWH without NAFLD, APRI and FIB-4 outperformed the other simple fibrosis biomarkers as first-tier tests. Our findings underline the need for considering liver disease in HIV mono-infected patients beyond NAFLD, especially in the presence of the unexplained elevation of liver transaminases.

We wish to acknowledge several limitations of this study. First, it was conducted at tertiary-care referral centers, and so the prevalence of significant liver fibrosis may be higher than in primary care settings. Secondly, liver biopsy, the gold standard to diagnose NAFLD and stage liver fibrosis, was unavailable. However, the EACS guidelines recommend noninvasive tests as initial diagnostic tools, as liver biopsy is not recommended for screening and routine clinical use [17].

\section{Conclusions}

A two-tier care pathway using fibrosis biomarkers as first-tiers could save up to $86.3 \%$ of TE examinations and care costs, helping personalized medicine and resource optimization in HIV clinics and low-resource settings. Similar to NAFLD in the HIVuninfected population, APRI and FIB-4 seem the most promising first-tier tests. This pathway could help the case-finding of at-risk $\mathrm{PWH}$ who will benefit from specialist referral for secondary fibrosis assessment, such as TE examination. The identification of $\mathrm{PWH}$ at a high risk for liver fibrosis is of paramount importance considering that there is no pharmacologic treatment for NAFLD-associated liver fibrosis and that PWH are excluded from most ongoing global clinical trials for NASH [53]. Future studies should focus on the longitudinal and long-term effects of the large-scale application of these models of care.

Author Contributions: G.S. contributed to the conception, study design, data and interpretation of the data, statistical analysis and first draft of the manuscript. J.M. and G.G. contributed to the study design, data and interpretation of the data and first draft of the manuscript. A.C. (Adriana Cervo), T.K., D.K., A.S.A.H., B.L., P.W., M.D., C.G., A.C. (Antonio Cascio) and G.M. contributed to data and interpretation of data. S.S. contributed to the study design and interpretation of data. All authors have read and agreed to the published version of the manuscript. 
Funding: G.S. is supported by a Senior Salary Award from Fonds de recherche du Québec-Santé (FRQS) (\#296306).

Institutional Review Board Statement: The Research Ethics Board of the Research Institute of MUHC (study code 14-182-BMD), MHMC (study code 254/12) and the Ethics Committee of the "Paolo Giaccone" University Hospital (study code v.1.05.1.18) approved the study, which was conducted according to the Declaration of Helsinki.

Informed Consent Statement: Informed consent was obtained from all subjects involved in the study.

Data Availability Statement: Data sharing statement: According to stipulations of the patient consent form signed by all study participants, the ethical restrictions imposed by our Institutional Ethics review boards (Institutional Ethics Review Board Biomedical B Research Ethics Board of the McGill University Health Centre), and the legal restrictions imposed by Canadian law regarding clinical trials, anonymized data are available upon request. Please send data access requests to Sheldon Levy, Biomedical B (BMB) Research Ethics Board (REB) Coordinator Centre for Applied Ethics, 5100, boul. de Maisonneuve Ouest, 5th floor, Office 576, Montréal, Québec, H4A 3T2, Canada.

Acknowledgments: Part of this work has been presented at the Liver Meeting of the American Association for the Study of Liver Diseases 2021.

Conflicts of Interest: G.S. has acted as speaker for Pfizer, Merck, Novonordisk, Novartis, Gilead, and AbbVie; served as an advisory board member for Merck, Gilead, Pfizer, Allergan, Novonordisk, Intercept, and Novartis; and has received research funding from Merck and Theratec. B.L. has acted as a speaker and advisory board member for ViiV, Gilead, and Merck, and received research funding from ViiV, Merck, and Gilead. PW has acted as a consultant for BMS, Gilead, Merck, and Novartis. M.D. has served as an advisory board member for Merck, Janssen, and Gilead. G.G. has acted as speaker for Merck, Gilead, and ViiV; served as an advisory board member for Merck and ViiV, and has received research funding from Merck, Gilead and ViiV. J.M., A.C. (Adriana Cervo), S.S., T.K., D.K., A.S.A.H, C.G. and A.C. (Antonio Cascio) have no conflict of interest to disclose.

\section{References}

1. Rinella, M.; Charlton, M. The globalization of nonalcoholic fatty liver disease: Prevalence and impact on world health. Hepatology 2016, 64, 19-22. [CrossRef] [PubMed]

2. Cai, J.; Osikowicz, M.; Sebastiani, G. Clinical significance of elevated liver transaminases in HIV-infected patients. AIDS 2019, 33, 1267-1282. [CrossRef] [PubMed]

3. Lemoine, M.; Serfaty, L.; Capeau, J. From nonalcoholic fatty liver to nonalcoholic steatohepatitis and cirrhosis in HIV-infected patients: Diagnosis and management. Curr. Opin. Infect. Dis. 2012, 25, 10-16. [CrossRef]

4. $\quad$ Maurice, J.B.; Patel, A.; Scott, A.J.; Patel, K.; Thursz, M.; Lemoine, M. Prevalence and risk factors of nonalcoholic fatty liver disease in HIV-monoinfection. AIDS 2017, 31, 1621-1632. [CrossRef] [PubMed]

5. Sterling, R.K.; Smith, P.G.; Brunt, E.M. Hepatic steatosis in human immunodeficiency virus: A prospective study in patients without viral hepatitis, diabetes, or alcohol abuse. J. Clin. Gastroenterol. 2013, 47, 182-187. [CrossRef] [PubMed]

6. Crum-Cianflone, N.; Dilay, A.; Collins, G.; Asher, D.; Campin, R.; Medina, S.; Goodman, Z.; Parker, R.; Lifson, A.; Capozza, T.; et al. Nonalcoholic fatty liver disease among HIV-infected persons. J. Acquir. Immune Defic. Syndr. 2009, 50, 464-473. [CrossRef]

7. Vuille-Lessard, E.; Lebouche, B.; Lennox, L.; Routy, J.P.; Costiniuk, C.T.; Pexos, C.; Giannakis, A.; Szabo, J.; Klein, M.B.; Sebastiani, G. Nonalcoholic fatty liver disease diagnosed by transient elastography with controlled attenuation parameter in unselected HIV monoinfected patients. AIDS 2016, 30, 2635-2643.

8. Guaraldi, G.; Lonardo, A.; Maia, L.; Palella, F.J., Jr. Metabolic concerns in aging HIV-infected persons: From serum lipid phenotype to fatty liver. AIDS 2017, 31 (Suppl. 2), S147-S156. [CrossRef]

9. Rockstroh, J.K.; Mohr, R.; Behrens, G.; Spengler, U. Liver fibrosis in HIV: Which role does HIV itself, long-term drug toxicities and metabolic changes play? Curr. Opin. HIV AIDS 2014, 9, 365-370. [CrossRef]

10. Pembroke, T.; Deschenes, M.; Lebouche, B.; Benmassaoud, A.; Sewitch, M.; Ghali, P.; Wong, P.; Halme, A.; Vuille-Lessard, E.; Pexos, C.; et al. Hepatic steatosis progresses faster in HIV mono-infected than HIV /HCV co-infected patients and is associated with liver fibrosis. J. Hepatol. 2017, 67, 801-808. [CrossRef]

11. Vodkin, I.; Valasek, M.A.; Bettencourt, R.; Cachay, E.; Loomba, R. Clinical, biochemical and histological differences between HIV-associated NAFLD and primary NAFLD: A case-control study. Aliment. Pharm. 2015, 41, 368-378. [CrossRef] [PubMed]

12. Lemoine, M.; Lacombe, K.; Bastard, J.P.; Sebire, M.; Fonquernie, L.; Valin, N.; Fellahi, S.; Capeau, J.; Girard, P.M.; Meynard, J.L. Metabolic syndrome and obesity are the cornerstones of liver fibrosis in HIV-monoinfected patients: Results of the METAFIB study. AIDS 2017, 31, 1955-1964. [CrossRef] [PubMed] 
13. Benmassaoud, A.; Ghali, P.; Cox, J.; Wong, P.; Szabo, J.; Deschenes, M.; Osikowicz, M.; Lebouche, B.; Klein, M.B.; Sebastiani, G. Screening for nonalcoholic steatohepatitis by using cytokeratin 18 and transient elastography in HIV mono-infection. PLoS ONE 2018, 13, e0191985. [CrossRef]

14. Price, J.C.; Seaberg, E.C.; Badri, S.; Witt, M.D.; D'Acunto, K.; Thio, C.L. HIV monoinfection is associated with increased aspartate aminotransferase-to-platelet ratio index, a surrogate marker for hepatic fibrosis. J. Infect. Dis. 2012, 205, 1005-1013. [CrossRef]

15. Younossi, Z.M.; Koenig, A.B.; Abdelatif, D.; Fazel, Y.; Henry, L.; Wymer, M. Global epidemiology of nonalcoholic fatty liver disease-Meta-analytic assessment of prevalence, incidence, and outcomes. Hepatology 2016, 64, 73-84. [CrossRef]

16. Taylor, R.S.; Taylor, R.J.; Bayliss, S.; Hagstrom, H.; Nasr, P.; Schattenberg, J.M.; Ishigami, M.; Toyoda, H.; Wong, V.W.-S.; Peleg, N.; et al. Association Between Fibrosis Stage and Outcomes of Patients With Nonalcoholic Fatty Liver Disease: A Systematic Review and Meta-Analysis. Gastroenterology 2020, 158, 1611-1625.e1612. [CrossRef]

17. Ryom, L.; Cotter, A.; De Miguel, R.; Beguelin, C.; Podlekareva, D.; Arribas, J.R.; Marzolini, C.; Mallon, P.; Rauch, A.; Kirk, O.; et al 2019 update of the European AIDS Clinical Society Guidelines for treatment of people living with HIV version 10.0. HIV Med. 2020, 21, 617-624. [CrossRef]

18. Introduction: Standards of Medical Care in Diabetes-2019. Diabetes Care 2019, 42 (Suppl. 1), S1-S2. [CrossRef]

19. European Association for the Study of The Liver; European Association for the Study of Diabetes. EASL-EASD-EASO Clinical Practice Guidelines for the management of non-alcoholic fatty liver disease. J. Hepatol. 2016, 64, 1388-1402. [CrossRef]

20. Cervo, A.; Shengir, M.; Patel, K.; Sebastiani, G. NASH in HIV. Curr. HIV/AIDS Rep. 2020, 17, 601-614.

21. Davyduke, T.; Tandon, P.; Al-Karaghouli, M.; Abraldes, J.G.; Ma, M. Impact of Implementing a "FIB-4 First" Strategy on a Pathway for Patients With NAFLD Referred From Primary Care. Hepatol. Commun. 2019, 3, 1322-1333. [CrossRef] [PubMed]

22. Shaheen, A.A.; Riazi, K.; Medellin, A.; Bhayana, D.; Kaplan, G.G.; Jiang, J.; Park, R.; Schaufert, W.; Burak, K.W.; Sargious, M.; et al. Risk stratification of patients with nonalcoholic fatty liver disease using a case identification pathway in primary care: A cross-sectional study. CMAJ Open 2020, 8, E370-E376. [CrossRef]

23. Srivastava, A.; Gailer, R.; Tanwar, S.; Trembling, P.; Parkes, J.; Rodger, A.; Suri, D.; Thorburn, D.; Sennett, K.; Morgan, S.; et al. Prospective evaluation of a primary care referral pathway for patients with non-alcoholic fatty liver disease. J. Hepatol. 2019, 71, 371-378. [CrossRef] [PubMed]

24. McPherson, S.; Stewart, S.F.; Henderson, E.; Burt, A.D.; Day, C.P. Simple non-invasive fibrosis scoring systems can reliably exclude advanced fibrosis in patients with non-alcoholic fatty liver disease. Gut 2010, 59, 1265-1269. [CrossRef]

25. Angulo, P.; Bugianesi, E.; Bjornsson, E.S.; Charatcharoenwitthaya, P.; Mills, P.R.; Barrera, F.; Haflidadottir, S.; Day, C.P.; George, J. Simple noninvasive systems predict long-term outcomes of patients with nonalcoholic fatty liver disease. Gastroenterology 2013, 145, 782-789.e784. [CrossRef]

26. Guaraldi, G.; Orlando, G.; Zona, S.; Menozzi, M.; Carli, F.; Garlassi, E.; Berti, A.; Rossi, E.; Roverato, A.; Palella, F. Premature age-related comorbidities among HIV-infected persons compared with the general population. Clin. Infect. Dis 2011, 53, 1120-1126. [CrossRef] [PubMed]

27. Guaraldi, G.; Squillace, N.; Stentarelli, C.; Orlando, G.; D'Amico, R.; Ligabue, G.; Fiocchi, F.; Zona, S.; Loria, P.; Esposito, R.; et al. Nonalcoholic fatty liver disease in HIV-infected patients referred to a metabolic clinic: Prevalence, characteristics, and predictors. Clin. Infect. Dis. Off. Publ. Infect. Dis. Soc. Am. 2008, 47, 250-257. [CrossRef]

28. Sebastiani, G.; Cocciolillo, S.; Mazzola, G.; Malagoli, A.; Falutz, J.; Cervo, A.; Petta, S.; Pembroke, T.; Ghali, P.; Besutti, G.; et al. Application of guidelines for the management of nonalcoholic fatty liver disease in three prospective cohorts of HIV-monoinfected patients. HIV Med. 2020, 21, 96-108. [CrossRef]

29. Castera, L. Noninvasive methods to assess liver disease in patients with hepatitis B or C. Gastroenterology 2012, 142, 1293-1302 e1294. [CrossRef]

30. Eddowes, P.J.; Sasso, M.; Allison, M.; Tsochatzis, E.; Anstee, Q.M.; Sheridan, D.; Guha, I.N.; Cobbold, J.F.; Deeks, J.J.; Paradis, V.; et al. Accuracy of FibroScan Controlled Attenuation Parameter and Liver Stiffness Measurement in Assessing Steatosis and Fibrosis in Patients With Nonalcoholic Fatty Liver Disease. Gastroenterology 2019, 156, 1717-1730. [CrossRef]

31. Lemoine, M.; Assoumou, L.; De Wit, S.; Girard, P.M.; Valantin, M.A.; Katlama, C.; Necsoi, C.; Campa, P.; Huefner, A.D.; Schulze Zur Wiesch, J.; et al. Diagnostic Accuracy of Noninvasive Markers of Steatosis, NASH, and Liver Fibrosis in HIV-Monoinfected Individuals at Risk of Nonalcoholic Fatty Liver Disease (NAFLD): Results From the ECHAM Study. J. Acquir. Immune Defic. Syndr. 2019, 80, e86-e94. [CrossRef] [PubMed]

32. Kleiner, D.E.; Brunt, E.M.; Van Natta, M.; Behling, C.; Contos, M.J.; Cummings, O.W.; Ferrell, L.D.; Liu, Y.C.; Torbenson, M.S.; Unalp-Arida, A.; et al. Design and validation of a histological scoring system for nonalcoholic fatty liver disease. Hepatology $\mathbf{2 0 0 5}$ 41, 1313-1321. [CrossRef] [PubMed]

33. Karlas, T.; Petroff, D.; Sasso, M.; Fan, J.G.; Mi, Y.Q.; de Ledinghen, V.; Kumar, M.; Lupsor-Platon, M.; Han, K.H.; Cardoso, A.C.; et al. Individual patient data meta-analysis of controlled attenuation parameter (CAP) technology for assessing steatosis. J. Hepatol. 2017, 66, 1022-1030. [CrossRef] [PubMed]

34. Williams, A.L.; Hoofnagle, J.H. Ratio of serum aspartate to alanine aminotransferase in chronic hepatitis. Relationship to cirrhosis. Gastroenterology 1988, 95, 734-739. [CrossRef]

35. Sattar, N.; Forrest, E.; Preiss, D. Non-alcoholic fatty liver disease. BMJ 2014, 349, g4596. [CrossRef] [PubMed] 
36. Wai, C.T.; Greenson, J.K.; Fontana, R.J.; Kalbfleisch, J.D.; Marrero, J.A.; Conjeevaram, H.S.; Lok, A.S. A simple noninvasive index can predict both significant fibrosis and cirrhosis in patients with chronic hepatitis C. Hepatology 2003, 38, 518-526. [CrossRef] [PubMed]

37. Vallet-Pichard, A.; Mallet, V.; Nalpas, B.; Verkarre, V.; Nalpas, A.; Dhalluin-Venier, V.; Fontaine, H.; Pol, S. FIB-4: An inexpensive and accurate marker of fibrosis in HCV infection. comparison with liver biopsy and fibrotest. Hepatology 2007, 46, 32-36. [CrossRef]

38. Harrison, S.A.; Oliver, D.; Arnold, H.L.; Gogia, S.; Neuschwander-Tetri, B.A. Development and validation of a simple NAFLD clinical scoring system for identifying patients without advanced disease. Gut 2008, 57, 1441-1447. [CrossRef]

39. Angulo, P.; Hui, J.M.; Marchesini, G.; Bugianesi, E.; George, J.; Farrell, G.C.; Enders, F.; Saksena, S.; Burt, A.D.; Bida, J.P.; et al. The NAFLD fibrosis score: A noninvasive system that identifies liver fibrosis in patients with NAFLD. Hepatology 2007, 45, 846-854. [CrossRef]

40. Congly, S.E.; Shaheen, A.A.; Swain, M.G. Modelling the cost effectiveness of non-alcoholic fatty liver disease risk stratification strategies in the community setting. PLoS ONE 2021, 16, e0251741. [CrossRef]

41. Ma, I.; Lau, C.K.; Ramdas, Z.; Jackson, R.; Naugler, C. Estimated costs of 51 commonly ordered laboratory tests in Canada. Clin. Biochem 2019, 65, 58-60. [CrossRef]

42. Ingiliz, P.; Valantin, M.A.; Duvivier, C.; Medja, F.; Dominguez, S.; Charlotte, F.; Tubiana, R.; Poynard, T.; Katlama, C.; Lombes, A.; et al. Liver damage underlying unexplained transaminase elevation in human immunodeficiency virus-1 mono-infected patients on antiretroviral therapy. Hepatology 2009, 49, 436-442. [CrossRef] [PubMed]

43. Lui, G.; Wong, V.W.; Wong, G.L.; Chu, W.C.; Wong, C.K.; Yung, I.M.; Wong, R.Y.; Yeung, S.L.; Yeung, D.K.; Cheung, C.S.; et al Liver fibrosis and fatty liver in Asian HIV-infected patients. Aliment. Pharm. 2016, 44, 411-421. [CrossRef] [PubMed]

44. Morse, C.G.; McLaughlin, M.; Matthews, L.; Proschan, M.; Thomas, F.; Gharib, A.M.; Abu-Asab, M.; Orenstein, A.; Engle, R.E.; $\mathrm{Hu}, \mathrm{X}$.; et al. Nonalcoholic Steatohepatitis and Hepatic Fibrosis in HIV-1-Monoinfected Adults with Elevated Aminotransferase Levels on Antiretroviral Therapy. Clin. Infect. Dis 2015, 60, 1569-1578. [CrossRef] [PubMed]

45. Lemoine, M.; Barbu, V.; Girard, P.M.; Kim, M.; Bastard, J.P.; Wendum, D.; Paye, F.; Housset, C.; Capeau, J.; Serfaty, L. Altered hepatic expression of SREBP-1 and PPARgamma is associated with liver injury in insulin-resistant lipodystrophic HIV-infected patients. AIDS 2006, 20, 387-395. [CrossRef] [PubMed]

46. Lombardi, R.; Lever, R.; Smith, C.; Marshall, N.; Rodger, A.; Bhagani, S.; Tsochatzis, E. Liver test abnormalities in patients with HIV mono-infection: Assessment with simple noninvasive fibrosis markers. Ann. Gastroenterol. 2017, 30, 349-356. [CrossRef] [PubMed]

47. Hussain, A.; Patel, P.J.; Rhodes, F.; Srivastava, A.; Patch, D.; Rosenberg, W. Decompensated cirrhosis is the commonest presentation for NAFLD patients undergoing liver transplant assessment. Clin. Med. (London) 2020, 20, 313-318. [CrossRef]

48. Kirkegaard-Klitbo, D.M.; Bendtsen, F.; Lundgren, J.; Nielsen, S.D.; Benfield, T. Group Cs: Poor Concordance between Liver Stiffness and Noninvasive Fibrosis Scores in HIV Infection without Viral Hepatitis. Clin. Gastroenterol. Hepatol. 2019, 18, 3049-3050 [CrossRef]

49. Lake, J.E.; Trevillyan, J. Impact of Integrase inhibitors and tenofovir alafenamide on weight gain in people with HIV. Curr. Opin. HIV AIDS 2021, 16, 148-151. [CrossRef] [PubMed]

50. Riazi, K.; Raman, M.; Taylor, L.; Swain, M.G.; Shaheen, A.A. Dietary Patterns and Components in Nonalcoholic Fatty Liver Disease (NAFLD): What Key Messages Can Health Care Providers Offer? Nutrients 2019, 11, 2878. [CrossRef]

51. Bischoff, J.; Gu, W.; Schwarze-Zander, C.; Boesecke, C.; Wasmuth, J.C.; van Bremen, K.; Dold, L.; Rockstroh, J.K.; Trebicka, J Stratifying the risk of NAFLD in patients with HIV under combination antiretroviral therapy (cART). EClinicalMedicine 2021, 40, 101116. [CrossRef] [PubMed]

52. Perazzo, H.; Cardoso, S.W.; Yanavich, C.; Nunes, E.P.; Morata, M.; Gorni, N.; da Silva, P.S.; Cardoso, C.; Almeida, C.; Luz, P.; et al. Predictive factors associated with liver fibrosis and steatosis by transient elastography in patients with HIV mono-infection under long-term combined antiretroviral therapy. J. Int. AIDS Soc. 2018, 21, e25201. [CrossRef] [PubMed]

53. Guaraldi, G.; Maurice, J.B.; Marzolini, C.; Monteith, K.; Milic, J.; Tsochatzis, E.; Bhagani, S.; Morse, C.G.; Price, J.C.; Ingiliz, P.; et al. New Drugs for NASH and HIV Infection: Great Expectations for a Great Need. Hepatology 2020, 71, 1831-1844. [CrossRef] [PubMed] 